\title{
Ultrasound-Guided Injection of Hyaluronic Gel Filler in the Temples: A Report of Two Cases
}

\author{
Patrick Micheels ${ }^{1, *}$, Stephanie Besse ${ }^{2}$, and Joan Vandeputte ${ }^{3}$ \\ ${ }^{1}$ Private practice, Geneva-Switzerland, 8, chemin de la Fontaine, 1224 Chêne-Bougeries- Switzerland \\ ${ }^{2}$ Institut privé de radiologie Medlmage, Geneva-Switzerland, 1, route de Florissant, 1206 Geneva- Switzerland \\ ${ }^{3}$ Private office, Oudernaerde- Belgium, Meerspoort 31, 9700 Oudenaarde Belgium
}

*Corresponding author: Micheels P, Private practice, Geneva-Switzerland, 8, chemin de la Fontaine, 1224 Chêne-Bougeries- Switzerland; Tel: + 4122 34711 13; E-mail: patrickscab@bluewin.ch

Received: 15 Oct, 2020 | Accepted: 28 Oct, 2020 | Published: 03 Nov, 2020

Citation: Micheels P, Besse S, Vandeputte J (2020) Ultrasound-Guided Injection of Hyaluronic Gel Filler in the Temples: A Report of Two Cases. J Clin Cosmet Dermatol 4(3): dx.doi.org/10.16966/2576-2826.156

Copyright: (C) 2020 Micheels P, et al. This is an open-access article distributed under the terms of the Creative Commons Attribution License, which permits unrestricted use, distribution, and reproduction in any medium, provided the original author and source are credited.

\begin{abstract}
Introduction: The aim of this case report was to assess the effect of injecting hyaluronic acid gel filler in the temporal fossa for the correction of skeletonisation in this specific anatomical area of the ageing face.
\end{abstract}

Subjects: Two white females agreed to take part in this study.

Materials and methodology: Under ultrasound guidance, we filled the temples with a hyaluronic acid gel (based on the patented "IPN-like" crosslinking technology developed by Laboratoires VIVACY, Archamps, France).

Results: Where the injection was carried out perfectly in contact with the bone, with the bevel of the needle pointed towards the bone, we observed a clinical improvement in the treated area. If injected a slightly more superficial into the muscle, the gel did not achieve a similar effect and it was not possible to observe its diffusion in the temporal muscle fibres by ultrasound. Adverse effects were limited to mild pain and discomfort for several hours. One of the subjects had a vice-like sensation for 48 to 72 hours following the injection.

Conclusion: In this ultrasound-guided study, we were able to observe the behaviour of hyaluronic acid gel filler injected into the temporal fossa in two subjects presenting with skeletonisation of the temporal area. To ensure optimal gel injection, the needle must be in contact with the bone with its bevel pointing downwards and the gel should be injected gently and slowly. This study requires to be validated on more subjects including a longer follow-up period.

Keywords: Cross-linked hyaluronic acid gel filler; Needle bevel; Temporal fossae

\section{Introduction}

In the context of anti-ageing management, we have previously described the behaviour of various Hyaluronic Acid (HA) gels following injection into the superficial, mid and deep reticular dermis [1-4]. We have also previously reported our observations regarding the subcutaneous injection of HA gel fillers, both ex vivo and in vivo [5-8]. In preparation for a lager study with planned longer-term follow-up, the main objective of this investigation was to observe the effects of different globally cohesive HA gel fillers following injection into the temporal fossa. Injections were carried out under ultrasound guidance using the same methodology described in our previous studies [1-8].

\section{Subjects}

Two white female patients (from PM's private aesthetic practice) agreed to participate in this investigation. Both women were provided with verbal and written information and allowed a two-week reflection period prior to signing an informed consent form, in line with the directives of the Helsinki declaration. Subjects 1 and 2 were 46 and 49.5 years old respectively. Both women had type III skin based on Fitzpatrick's classification [9] and their degree of photoageing was II according to the Glogau scale [10].

\section{Equipment}

Following consultation with Laboratoires Vivacy, Archamps, France, we selected HA gels with patented "IPN-like" (InterPenetreting Network Like ${ }^{\circ}$ ) cross-linking technology. We used Stylage $\mathrm{XL}^{\oplus}$ without lidocaine, batch No. EXI19038H 2021-07 and Stylage $\mathrm{XL}^{\oplus}$ with lidocaine, batch No. LXE18291F 2021-03 for subjects 1 and 2 respectively. A 23G1/4 needle, provided in the packaging, was used for injection. Injections were performed under ultrasound guidance at the MedImage Institute, Geneva, Switzerland using a SAMSUNG "RS80A Prestige" ultrasound 
machine with a high resolution (4-18 Mhz) probe from Méditron, Gland, Switzerland.

\section{Procedure}

After double disinfection of the skin with a non-alcoholic chlorexidine solution (Hybidil ${ }^{\circledR}$, CPS Cito Pharma Services GmbH, 8610 Uster, Switzerland), the needle was inserted perpendicular to the skin, $1 \mathrm{~cm}$ from the bony orbit in the cranial direction and one centimetre outward from the temporal crest into the temporal muscle until it came in contact with the bone (Figure 1). The ultrasound probe was then put in place after application of an aqueous ultrasound transmission gel.

In case of subject 1 the needle was not seen on ultrasound following insertion and the needle had to be bent and re-inserted, still perpendicular to the skin. Once in contact with bone, the syringe and needle were later tilted to facilitate placement of the ultrasound probe above the needle and its visualization on ultrasound (Figures 2 and 3 ).

A total of $0.5 \mathrm{ml} \mathrm{HA}$ gel was injected in each temporal fossa. The injection was video-recorded on ultrasound and images were taken before and after each injection. It should be noted that the use of realtime ultrasound meant that the procedure had to be performed in a semi-dark room. Furthermore, we did not have the same freedom of movement as in our normal clinical practice because it was not possible to freely move the examination table or to move around it this resulted in some of the injections being carried out with the operator's non-dominant hand.

\section{Results}

The operator, who is right handed, was optimally positioned during the filling of the right temporal fossa in both subjects, despite the presence of the ultrasound probe located above and in the axis of the needle. However, the situation was not the same for the left temporal fossa due to the need to use the non-dominant hand for injection as a result of the aforementioned constraints of the surroundings. The ultrasound results of the right and left temporal fossae filling in both subjects are presented in table 1 .

Subject 1, who received the gel without lidocaine, experienced pain, which she rated as 3/10 during and just after the injection. She reported that the character of the pain changed to "heaviness" 15 minutes after the injection, which spontaneously resolved after 30 minutes. She had a small haematoma on the right side over the injection site. Due to the need to reinsert the needle in this case to facilitate ultrasound visualisation, we believe that the haematoma was a consequence of the trauma to the skin and subcutaneous tissues rather than the HA gel (Figures 4 and 5).
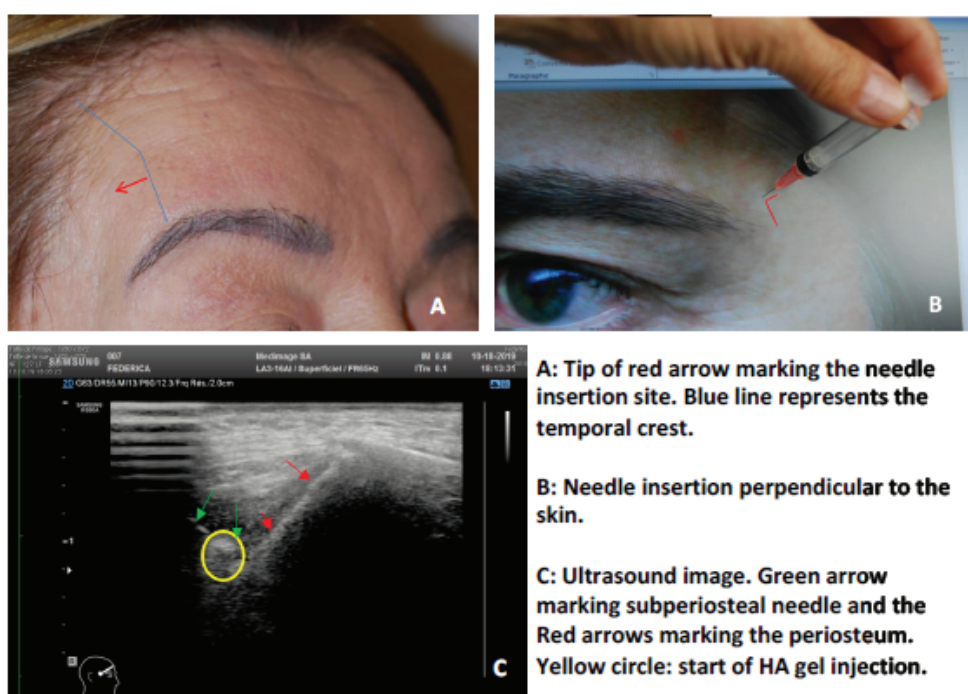

A: Tip of red arrow marking the needle insertion site. Blue line represents the temporal crest.

B: Needle insertion perpendicular to the skin.

C: Ultrasound image. Green arrow marking subperiosteal needle and the Red arrows marking the periosteum. Yellow circle: start of HA gel injection.

Figure 1: Needle insertion and tissue position.
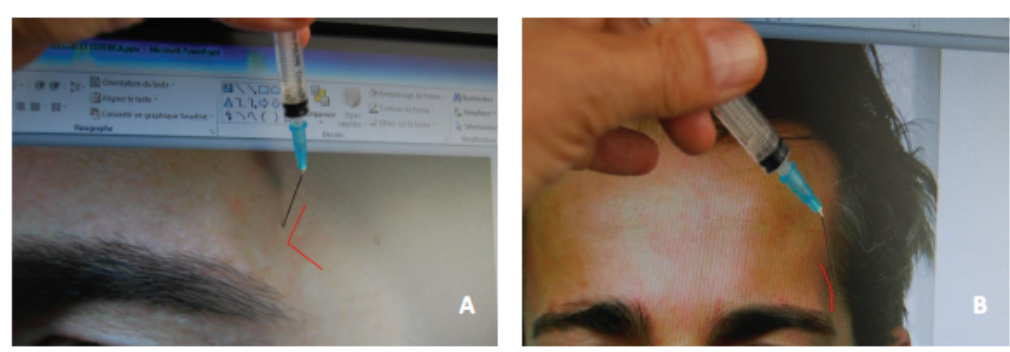

A: $90^{\circ}$ skin insertion of bent needle B: Tilt of syringe and needle once in contact with bone

Figure 2: Simulation of the needle insertion to facilitate ultrasound visualisation. 

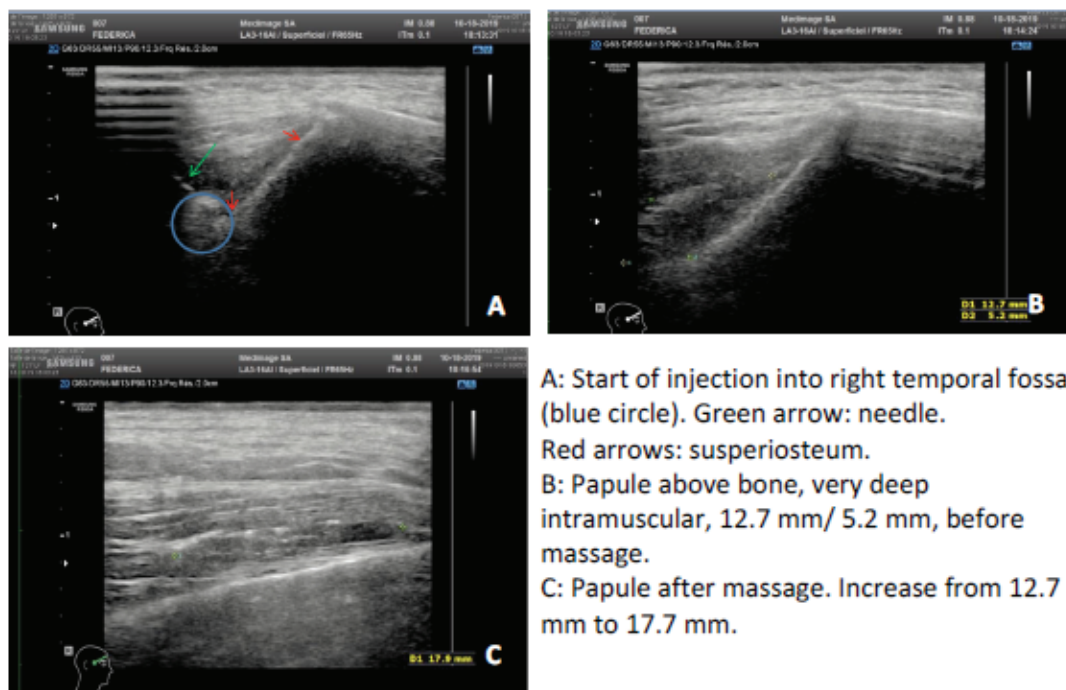

A: Start of injection into right temporal fossa (blue circle). Green arrow: needle.

Red arrows: susperiosteum.

B: Papule above bone, very deep

intramuscular, $12.7 \mathrm{~mm} / 5.2 \mathrm{~mm}$, before

massage.

C: Papule after massage. Increase from 12.7

$\mathrm{mm}$ to $17.7 \mathrm{~mm}$.

Figure 3: HA gel injection in Subject 1.

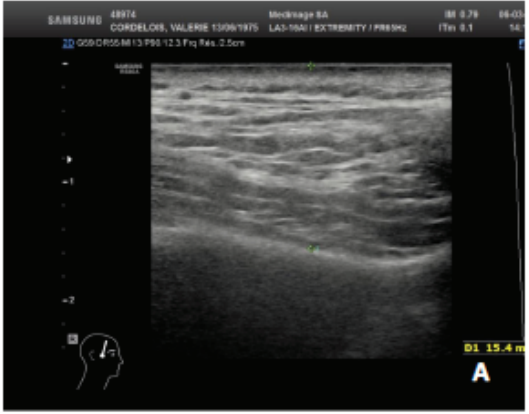

A: Ultrasound of right temporal region before needle insertion

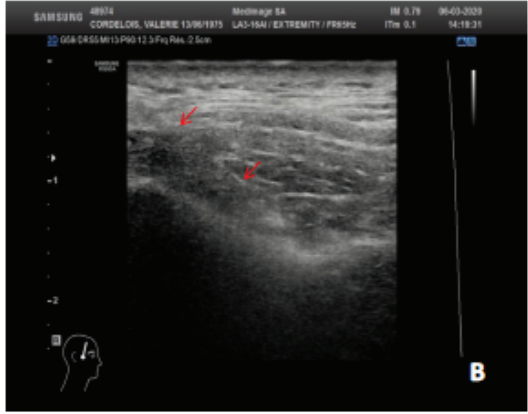

B: After needle insertion (red arrows) and before injection.

Figure 4: Needle insertion in subject 2-Right temporal fossa.

Subject 2 was surprised by the contact with the bone and reported a sensation of brisk pain, which she rated as $1 / 10$. After the injection, she reported a sensation of heaviness described to be similar to being given an anaesthetic at the dentist. This sensation lasted for 45 minutes following which she was asymptomatic. However, at home, the subject reported that she experienced a "vice-like" sensation mildly squeezing her skull for 48 to 72 hours. Of note, at the start of the injection, we observed some micro-bubbles of air during the filling of the right temporal fossa because the HA gel was not brought to the edge of the bevel of the needle prior to needle insertion (Table 1).

In the case of subject 1 , we did not take any pre and post injection photographs to assess clinical outcome because our focus was on the feasibility of performing the technique under ultrasound scan guidance. However, we managed to take pre-injection, immediately post injection and 2 months post injection for subject 2 (Figures 6 and 7). Immediately post injection a malar flush was noticed, probably due to the subject's stress and emotions. A noticeable improvement was observed in both temporal fossae immediately post injection (Figure 6). At two months, the result of the right side fossa was completely satisfactory, however, an element of under-correction was observed on the left side (Figure 7).

\section{Discussion}

In our opinion, the clinician's optimal operating position and comfort ensuring perfect alignment of their hand with the injection site are crucial when filling the temporal fossa to ensure the gel is correctly injected into the deep intramuscular area against the bone. In our preliminary observation, we were surprised that the injections in the left temporal fossa were more superficial than anticipated despite the perception that the needle was in contact with the bone. As stated above, this is probably a reflection of the suboptimal technical skill secondary to the use of the non-dominant hand for needle insertion and injection. It is imperative to ensure good contact with bone, an issue that the patient should be well informed about prior to the procedure emphasising what they might feel. Indeed, a more superficial injection of the same gel volume in an extremely mobile environment, as in the case of muscle fibres, seems to have a less marked clinical effect.

One of the strengths of our preliminary report is our ability to monitor the needle position and the HA gel behaviour prior to and 


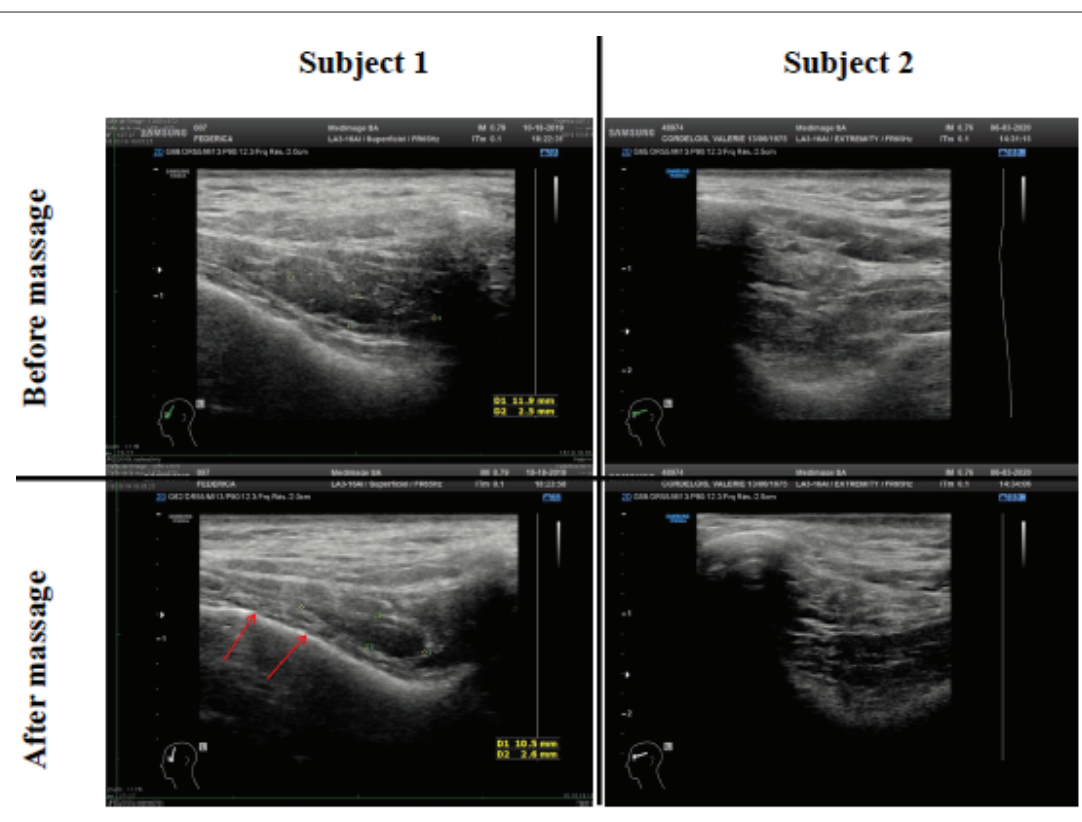

Figure 5: Ultrasound images of HA gel in left temporal fossa before and after massage.

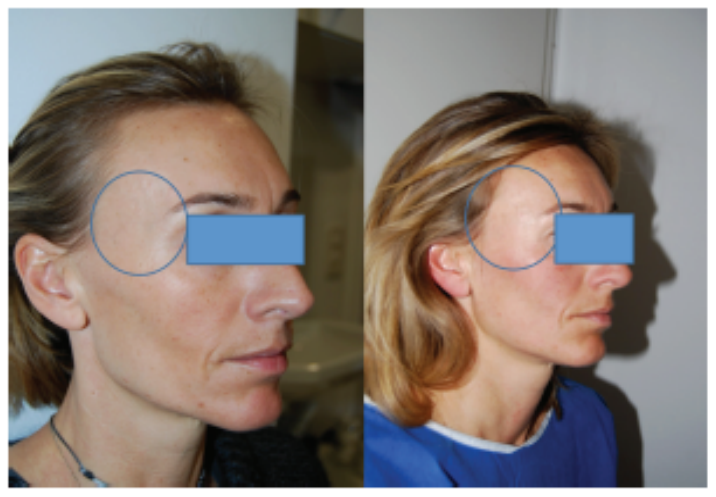

Before

After

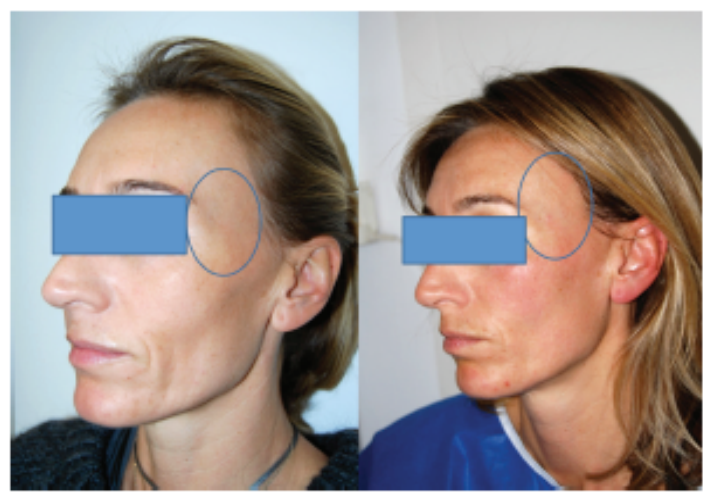

Before

After

Figure 6: Subject 2 before and immediately after HA gel injection.
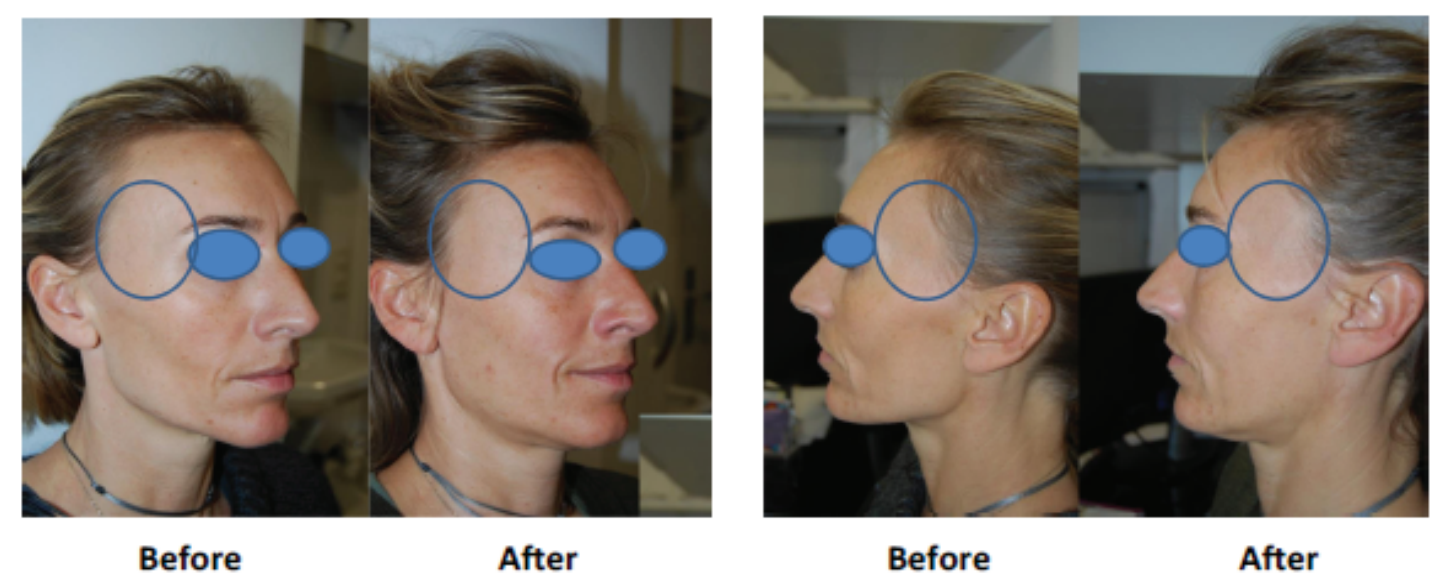

Before

After

Figure 7: Subject 2 before and 2 months after HA gel injection. 
Table 1: Ultrasound findings during and after filling.

\begin{tabular}{|c|c|c|}
\hline & Right temporal fossa & Left temporal fossa \\
\hline 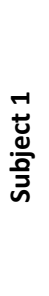 & $\begin{array}{l}\text { The position of the needle was perpendicular to and almost in } \\
\text { contact with the bone. The needle bevel pointed downwards. The } \\
\text { HA gel appeared as a convex papule, progressing in a fusiform } \\
\text { manner. It was hypoechogenic and homogeneous with regular } \\
\text { margins, there was no enhancement or posterior shadowing and } \\
\text { was pressing against the overlying tissues. After massage, the } \\
\text { papule thinned and lengthened appearing to partially } \\
\text { infiltrate the deep muscle fibres (Figure 3). }\end{array}$ & $\begin{array}{l}\text { The position of the needle was perpendicular to the bone but not } \\
\text { strictly in contact with it, probably due to the use of the non-dominant } \\
\text { hand. The bevel of the needle was pointing downwards. The HA gel } \\
\text { appeared as a fusiform, polylobed papule that did not come in contact } \\
\text { with the bone and visibly progressed into the muscle. The papule was } \\
\text { homogeneous and hypoechogenic with no posterior enhancement or } \\
\text { shadowing. It did not contain any air bubbles. After massage, the gel } \\
\text { appeared to spread and infiltrate in between muscle fibres. (Figure 5) }\end{array}$ \\
\hline 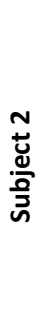 & $\begin{array}{l}\text { The position of the needle was slightly obliquely positioned to and } \\
\text { more distant from the bone, compared to Subject } 1 \text {. The bevel } \\
\text { appeared pointing upwards (Figure 4). The gel appeared in the form } \\
\text { of a round papule and did not come in contact with the bone, and } \\
\text { hence less deep. It was hypoechogenic, discreetly heterogeneous, } \\
\text { polycyclic with regular margins. The gel gradually progressed } \\
\text { superficially. Some micro-bubbles of air could be seen. The papule } \\
\text { had a clearly demarcated posterior enhancement. No gel diffusion } \\
\text { could be clearly seen after massage. }\end{array}$ & $\begin{array}{l}\text { The needle was in an oblique position to the bone and at some } \\
\text { distance, probably due to the use of the non-dominant hand for the } \\
\text { procedure. The bevel of the needle pointed upwards. The HA gel } \\
\text { appeared as a heterogeneous area, causing distortion of the muscle } \\
\text { fibres, with no clearly defined margins. There was no posterior } \\
\text { shadowing or enhancement. The gel progressed superficially between } \\
\text { the muscle fibres (Figure 5). Massage resulted in no changes to the } \\
\text { ultrasound image. }\end{array}$ \\
\hline
\end{tabular}

following injection respectively. We were particularly interested to note that even when the needle was in contact with the bone, the slightest movement, such as pressure from the ultrasound probe here, changed the position of the needle and thus the gel injection site. It is important to highlight that if the gel spreads rapidly and only upwards, towards the superficial muscle layers, there is a risk of significant dispersion of the gel, making it difficult to follow its progression using ultrasound. Furthermore, this can be associated with a suboptimal aesthetic result.

Following the analysis of our subjects' response to the HA gel injections, the obtained ultrasound scan images and clinical outcome, we suggest that the following points are critical for a successful procedure:

- We recommend the use a lidocaine containing HA gel filler.

- The gel should be brought to the bevel of the needle prior to insertion.

- Ensure the needle bevel is pointing downwards (i.e. towards the bone). In our opinion, this promotes the correct positioning of the gel.

- Proceed with needle insertion until true contact with the bone is achieved. It is good practice to forewarn the patient of what to expect because this can cause an odd sensation or even sharp pain.

- Inject the gel slowly.

- Massage the area following injection to ensure the homogeneous distribution of the gel in the temporal fossa.

- Avoid over-correction because under-correction can be finely adjust after three months.

\section{Conclusion}

We recognise that our report is preliminary with relatively shortterm follow-up, nonetheless, we were able to observe and document the coherent behaviour of needle injected HA gel filler in the temporal fossa before and after massage in two subjects presenting with skeletonisation of the temporal area. Addition of lidocaine to the HA gel during its production appears to help in reducing the pain experienced by patients undergoing such treatment.
To ensure bone proximity, the needle must be in contact with the bone with the bevel pointing downwards. The gel should be injected gently and slowly to mitigate the risk of pain and discomfort resulting from distension of overlying tissues. We observed that following these steps resulted in clinical improvement in the treated zone, even with a relatively small amount of HA gel.

We appreciate that increasing the sample size, duration of follow-up and use of more detailed imaging modalities are required to validate our findings. Such a study is currently on going in our centre to have a better understanding of the detailed behaviour of (IPN-Like ${ }^{\infty}$ Stylage ${ }^{\infty}$ $\mathrm{XL}) \mathrm{HA}$ gel for the management of skeletonisation of the temporal fossa.

\section{Conflicts of interest}

The hyaluronic acid gel injections were provided by Laboratoires VIVACY, Archamps, France. P. Micheels is a consultant and trainer for Allergan, Antéis-Merz, Galderma-Q-Med Suisse, IBSA, Merz, Teoxane and Vivacy. The ultrasound scans of the second subject were funded by Laboratoires VIVACY. J. Vandeputte is a consultant for Merz and Advanced Aesthetic Technologies. St. Besse declares no conflicts of interest.

\section{References}

1. Micheels P, Sarazin D, Besse S, Sundaram H, Flynn TC (2013) A Blanching Technique for Intradermal Injection of the Hyaluronic Acid Belotero. Plast Reconstr Surg 132: 59S-68S.

2. Tran C, Carraux P, Micheels P, Kaya G, Salomon D (2014) In vivo Bio-Integration of Three Hyaluronic Acid Fillers in Human Skin: A Histological Study. Dermatology 228: 47-54.

3. Micheels P, Besse S, Flynn TC, Sarazin D, Elbaz Y (2012) Superficial Dermal Injection of Hyaluronic Acid Soft Tissue Fillers: Comparative Ultrasound Study. Dermatol Surg 38: 1162-1169.

4. Micheels P, Besse S, Sarrazin D (2016) Visual, Ultrasonographic, and Microscopic Study on Hyaluronic Acid-Based Gel. J Drugs Dermatol 15: 1092-1098. 
5. Micheels P, Besse S, Sarazin D (2017) Two Crosslinking Technologies for Superficial Reticular Dermis Injection: A Comparative Ultrasound and Histologic Study. J Clin Aesthet Dermatol 10: 29-36.

6. Micheels P, Besse S, Sarazin D, Quinodoz P, Elias B, et al. (2017) Ultrasound and Histologic Examination after Subcutaneous Injection of Two Volumizing Hyaluronic Acid Fillers: A preliminary Study. Plast Reconstr Surg Glob Open 5: e1222.

7. Santer V, Molliard SG, Micheels P, Río-Sancho SD, Quinodoz P, et al (2019) Hyaluronic Acid After Subcutaneous Injection-An Objective Assessment. Dermatol Surg 45: 108-116.
8. Vandeputte J, Leemans G, Dhaene K, Forsyth R, Vanslembrouck J, et al (2020) Spreading Pattern and Tissue Response to Hyaluronic Acid Gel Injections in the Subcutis: Ultrasound Videos, Ultrasound Measurements, and Histology. Aesthet Surg J sjaa007.

9. Gupta V, Sharma VK (2019) Skin typing: Fitzpatrick grading and others. Clin Dermatol 37: 430-436.

10. Brannon HL (2020) How the Glogau Classification System Measures Photoaging. 